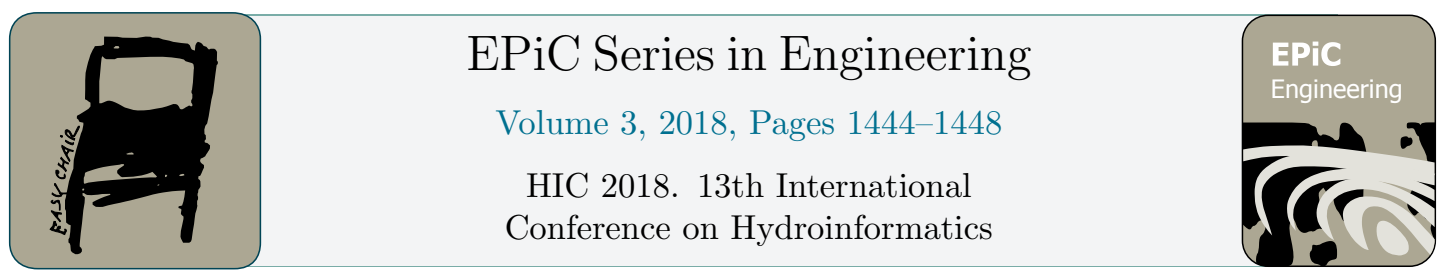

\title{
Large-Eddy Simulation of Open-Channel Flow with Rigid Submerged Vegetation
}

\author{
Alessandro Monti ${ }^{1}$, Mohammad Omidyeganeh $^{2}$, and Alfredo Pinelli ${ }^{3}$ \\ 1 City, University of London, London, U.K. \\ alessandro.monti@city.ac.uk \\ 2 City, University of London, London, U.K. \\ omid.yeganeh@city.ac.uk \\ 3 City, University of London, London, U.K. \\ alfredo.pinelli.1@city.ac.uk
}

\begin{abstract}
A large-eddy simulation over a fully submerged, rigid canopy in an open-channel flow under transitional canopy flow regime has been carried out. The simulations revealed a flow structure characterized by the emergence of coherent structures, which are very elongated in the streamwise direction occupying a large portion of the flow inside and outside the canopy. These very large structures have a strong impact on the flow inside the canopy as well.
\end{abstract}

\section{Introduction}

Filamentous structures embedded in fluid flows are ubiquitous in nature performing several, different tasks, from thermal regulation to water harvesting, from flow sensing to transport of particles. An interesting case is the one offered by vegetative plants (macrophytes) in river flows. Macrophytes affect significantly the stream controlling the sediment dynamics, creating habitats for microorganisms and cover for fish, also influencing the nutrient dynamics and improving water quality (especially relevant to the treatment of grey-waters) [5, 7]. These biological phenomena have been estimated to have an overall economic impact of over 10 trillion dollars in the globe [3]. The societal, technological and economic importance has driven a special interest in unravelling the mechanisms of the interaction between the vegetation and the flow. In particular, the flow over instream-submerged vegetation has received much attention over the last decades. In this condition, the flow-plants interaction triggers the development of large coherent structures that affect the scalar fluxes governing the nutrient exchange, sediment deposition, and the chemical reaction within the vegetative zones. Although the research on vegetative flows moved its first steps during the 1950s, relevant contributions on the characterization of the effects of the vegetative canopies in waterways were defined much more recently [8]. One of the most important contributions has been the introduction of a classification of canopy flows exclusively based on geometrical considerations. Specifically, the proposed categorization is based on two geometrical ratios. The first one involves the flow depth $H$ and

G. La Loggia, G. Freni, V. Puleo and M. De Marchis (eds.), HIC 2018 (EPiC Series in Engineering, vol. 3), 

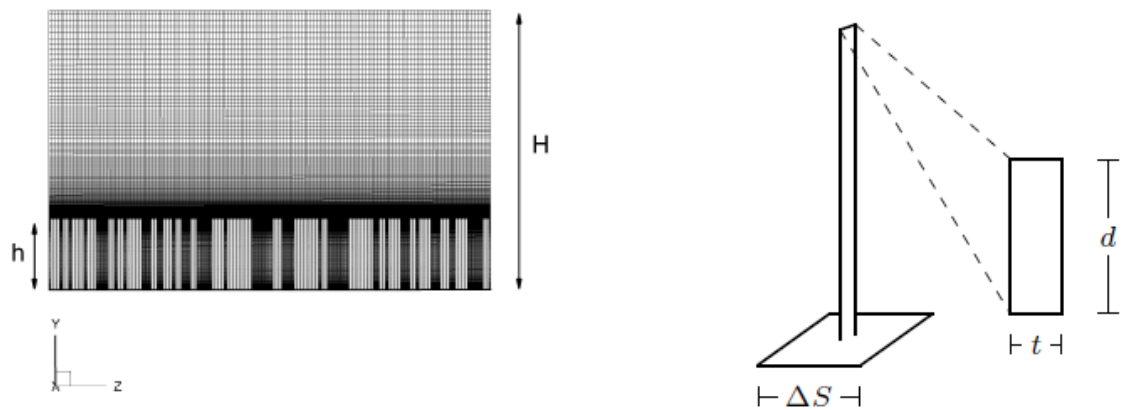

Figure 1: Geometrical parameters governing a canopy flows according to Nepf [8]. In our simulation, the filaments are randomly distributed on the canopy bed, each one occupying an average area $\Delta S^{2}$.

the canopy height $h$ (see Figure 1). If $H / h=1$, the flow is termed as emergent, otherwise if $1<H / h<5$ it is called shallow submerged and deeply submerged when $H / h>10$. The second geometric parameter introduces a specific classification for submerged canopy flows identifying two limiting behaviours that are approached as the canopy density varies. If the ratio between the frontal area and the bed area, defined as $\lambda=a h$, where $a=d / \Delta S^{2}$ (see Figure 1), is less than a threshold value (i.e. $\lambda<0.1$ ) then the fluid velocity within and above the canopy shows a behaviour comparable to the one observed in a turbulent boundary-layer over a rough wall. The sparse regime is also characterized by the dominance of bed drag over the actual canopy drag. For large values of $\lambda$ (i.e. $\lambda>0.1$ ), the canopy drag is larger than the one offered by the bed. Under this condition, termed as dense regime, the drag distribution displays a discontinuity at the top of the canopy that, in turns, determines the appearance of an inflection point in the velocity profile near the canopy edge. The present contribution makes use of a large-eddy simulation (LES) to characterize the behaviour of the flow over a submerged, mildly dense, rigid canopy. In particular, we pretend to explore and unravel the mechanisms of the interaction between the fluid flow and the dense rigid canopy by identifying the physical parameters that govern the mixing mechanisms within the different flow layers and by exploring the impact of the sweep/ejection events in the canopy edge.

\section{Methodology}

The present study has tackled the simulation of the turbulent flow field using an in-house developed incompressible Navier-Stoke solver (SUSA). The solver deals with the governing LES equations with a second order accurate, cell centred finite volume approach and a second order time accurate pressure correction scheme. Readers interested in the solver and on the extensive validation campaign can find detailed information in [9]. Further information on the ILSA model used for the subgrid stress closure can be found in [10]. Differently from other approaches, we resolve the canopy directly introducing a set of rigid and solid filaments of finite cross-section which are placed perpendicularly to the horizontal wall. To impose the boundary conditions that each rigid filament exerts on the fluid (i.e. zero velocity) we have used a particular immersed boundary (IB) method that, as many other IB methods, deals with immersed bodies using a set of Lagrangian nodes that, in general, do not conform with the underlying Cartesian grid 


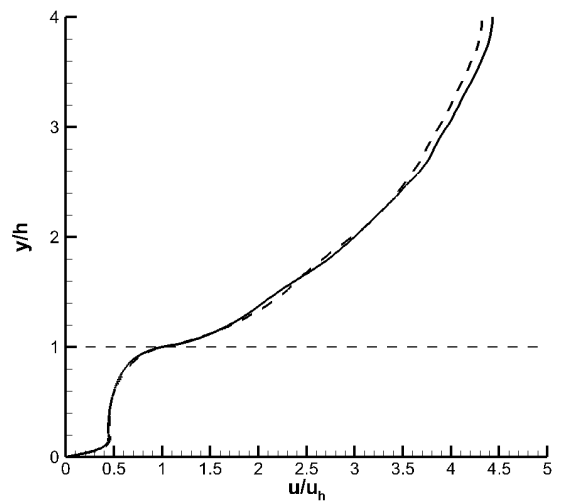

Figure 2: Method verification: mean velocity profile normalized with the value at the canopy tip, $u_{h}$. Solid line, reference method and dashed line, current method.

used to discretize the LES equations. Further information on the implementation of the IB method can be found in [4]. This approach allows significant computational savings since the filament cross section is kept into account with a single point. Other, more traditional, IB methods (e.g., direct forcing) would require tens of points for the imposition of the boundary value on the filament. The performance and validation of the methodology has been carried out in a small domain with four filaments that were treated with accurate, but expensive IB methods versus the present one (see Figure 2). For the present simulation, we have considered a periodic computational domain in stream- and spanwise directions. The size of the box in the streamwise, wall-normal, and spanwise directions is $L_{x}=8 \pi h, L_{y}=4 h$ and $L_{z}=6 \pi h$ (similar to [1]). At the bottom wall, a no-slip boundary condition is imposed while a free-slip condition is set at the top surface. The bulk Reynolds number is $\operatorname{Re}_{b}=U_{b} H / \nu=6000$ (where $U_{b}$ is the bulk velocity and $\nu$ is the kinematic viscosity), similar to experiments [6]. The Cartesian grid system is uniform in horizontal directions that yields a resolution in wall units (the friction velocity derived from the maximum total shear stress) of $\Delta x^{+} \approx 12, \Delta y_{h} \approx 0.3$ and $\Delta z^{+} \approx 12$, in the streamwise, wall-normal and spanwise directions respectively.

\section{Results and Discussion}

As reference case for a qualitative comparison of the obtained results, we have considered the work of Bailey \& Stoll [2]. They have numerically simulated a similar canopy flow configuration using LES and mimicking the presence of the filamentous layer with a set of distributed volume forces. Despite the differences in the approach chosen to model the presence of the canopy, the agreement between the present results and the ones reported in [2] is quite satisfactory. From Figure 3, that shows the mean velocity profile, we can note that at least three separate regions can be identified. The outermost one is characterized by a mean velocity shape that reminds of the turbulent boundary-layer one featuring a logarithmic region with a slope that does not match the canonical one (see Figure 3). Closer to the canopy edge, the mean velocity profile exhibits an inflection point typical of the transitional/dense canopies regimes (note that, following the classification in [8], our $\lambda \approx 0.35)$. The inflection point triggers a Kelvin-Helmholtz 

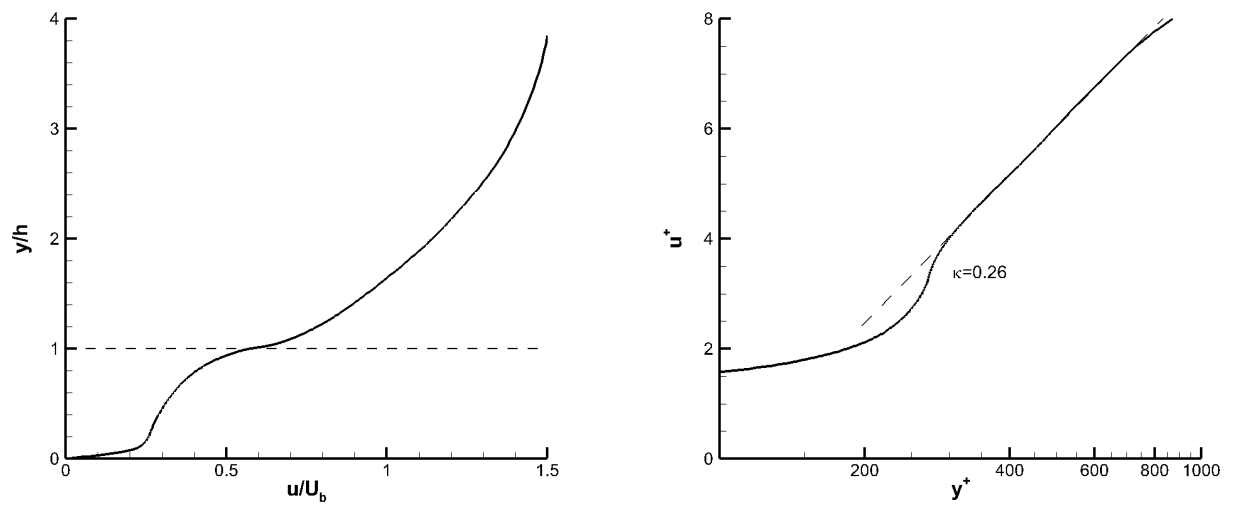

Figure 3: Mean velocity profile normalized with the bulk velocity $U_{b}$ (left) and the friction velocity $u_{f}$ (right).

instability and the eventual formation of large spanwise vortices that can be observed in Figure 4 (bottom left). Three-dimensional realization of the flow in Figure 4 illustrates the presence of large streamwise oriented vortices that occupy the whole depth of the channel and even penetrate the canopy and reach the bed (Figure 4 bottom right). These streamwise vortices are inclined toward the free surface at downstream due to presence of spanwise vortices (Figure 4 bottom left). The presence of large spanwise vortices that penetrates into the bed suggests that the canopy flow can be classified in a transitional state [8]. The highly defined resolution of the present work has also allowed to highlight new flow dynamics in which large and very long streamwise vortices co-exist in the flow. These very large structures deeply penetrate in the canopy and definitely have a strong influence in the canopy mixing (e.g., nutrient transport). In particular, in the present configuration, the large streamwise vortices have a spanwise size of about $2 h-3 h$ and therefore they are too large for being captured in narrow flume experiment $[6]$.

\section{References}

[1] B.N. Bailey and R. Stoll. Turbulence in sparse, organized vegetative canopies: a large-eddy simulation study. Boundary-Layer Meteorology, 147(3):369-400, 2013.

[2] B.N. Bailey and R. Stoll. The creation and evolution of coherent structures in plant canopy flows and their role in turbulent transport. Journal of Fluid Mechanics, 789:425-460, 2016.

[3] R. Costanza, R. d'Arge, R. De Groot, S. Farber, M. Grasso, B. Hannon, K. Limburg, S. Naeem, R.V. O'neill, J. Paruelo, et al. The value of the world's ecosystem services and natural capital (1997). The Globalization and Environment Reader, page 117, 2016.

[4] J. Favier, A. Revell, and A. Pinelli. A lattice boltzmann-immersed boundary method to simulate the fluid interaction with moving and slender flexible objects. Journal of Computational Physics, 261:145-161, 2014.

[5] M. Ghisalberti and H.M. Nepf. Mixing layers and coherent structures in vegetated aquatic flows. Journal of Geophysical Research: Oceans, 107(C2), 2002.

[6] M. Ghisalberti and H.M. Nepf. The limited growth of vegetated shear layers. Water Resources Research, 40(7), 2004. 

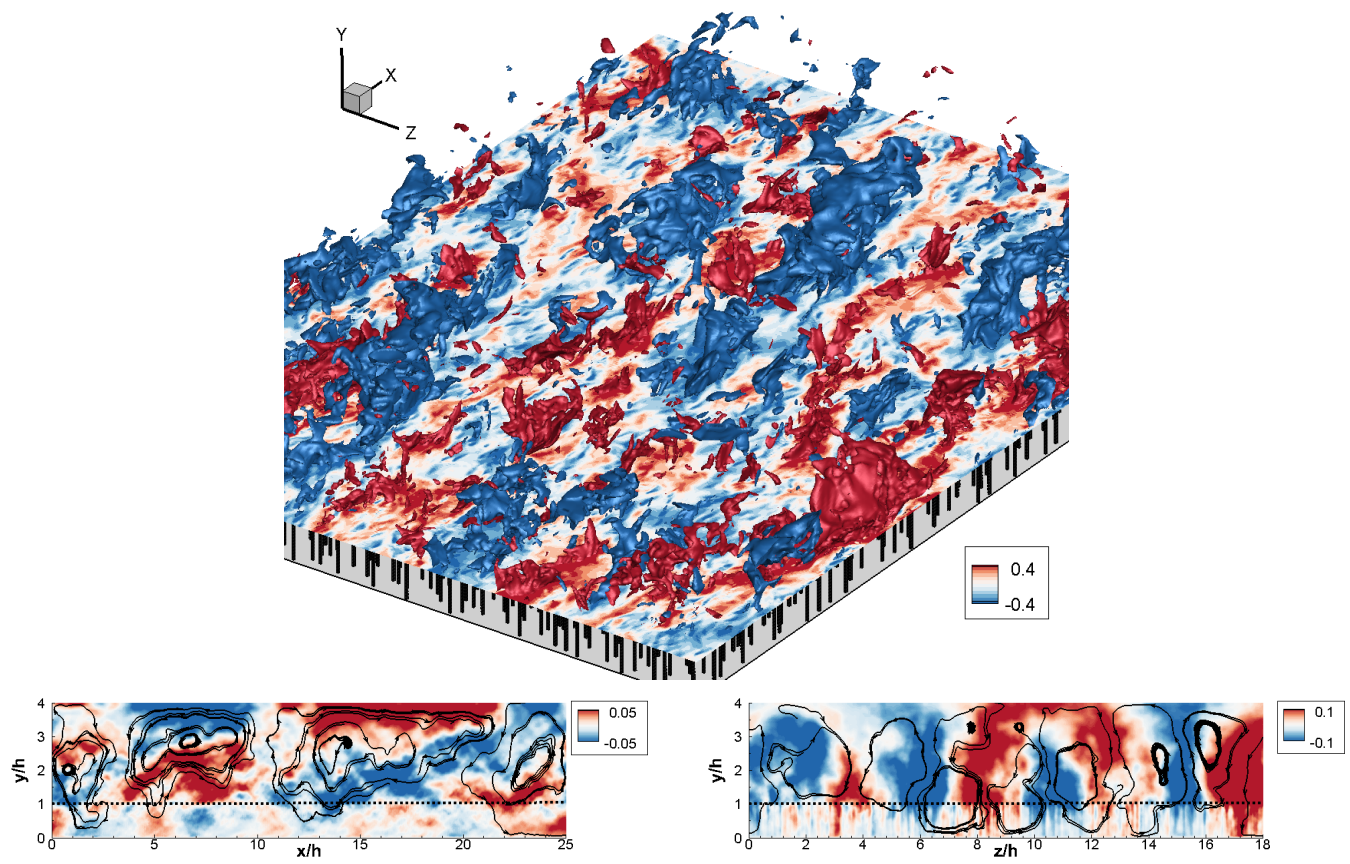

Figure 4: Top: filaments are shown by black stems; a horizontal plane is placed at the canopy edge to show the contours of the streamwise velocity fluctuations $u^{\prime} / U_{b}$, and, on top of it, the isosurfaces of streamwise velocity fluctuations are illustrated. Bottom left: contours of streamwise velocity fluctuations averaged in spanwise direction, $\left\langle u^{\prime}\right\rangle_{z} / U_{b}$. Bottom right: contours of streamwise velocity fluctuations averaged in streamwise direction, $\left\langle u^{\prime}\right\rangle_{x} / U_{b}$. The dotted line represents the canopy edge and the streamlines represent the in-plane fluctuations field averaged in the normal direction to the plane.

[7] R. Mars, K. Mathew, and G. Ho. The role of the submergent macrophyte triglochin huegelii in domestic greywater treatment. Ecological Engineering, 12(1):57-66, 1999.

[8] H.M. Nepf. Flow and transport in regions with aquatic vegetation. Annual Review of Fluid Mechanics, 44:123-142, 2012.

[9] M. Omidyeganeh and U. Piomelli. Large-eddy simulation of three-dimensional dunes in a steady, unidirectional flow. part 2. flow structures. Journal of Fluid Mechanics, 734:509-534, 2013.

[10] A. Rouhi, U. Piomelli, and B.J. Geurts. Dynamic subfilter-scale stress model for large-eddy simulations. Physical Review Fluids, 1(4):044401, 2016. 